ORNL/TM-2013/151

National Center for Computatational Sciences

\title{
Ceph Parallel File System Evaluation Report
}

\author{
Feiyi Wang Mark Nelson \\ ORNL/NCCS Inktank Inc. \\ Other contributors: \\ Sarp Oral \\ Doug Fuller \\ Scott Atchley \\ Blake Caldwell \\ James Simmons \\ Brad Settlemyer \\ Jason Hill \\ Sage Weil (Inktank Inc.) \\ Prepared by \\ OAK RIDGE NATIONAL LABORATORY \\ Oak Ridge, Tennessee 37831-6283 \\ managed by \\ UT-BATTELLE, LLC \\ for the \\ U.S. DEPARTMENT OF ENERGY
}

This research was supported by, and used the resources of, the Oak Ridge Leadership Computing Facility, located in the National Center for Computational Sciences at ORNL, which is managed by UT Battelle, LLC for the U.S. DOE (under the contract No. DE-ACo5-0oOR22725). 


\section{Contents}

1 Introduction 1

2 Testbed Environment Description 2

3 Baseline Performance 3

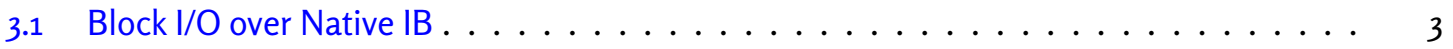

3.2 IP over IB ........................... 3

4 System Tuning 3

5 XFS Performance As Backend File System 4

6 Ceph RADOS Scaling: Initial Test

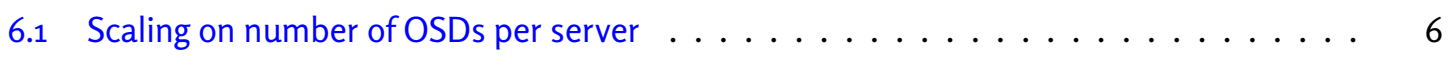

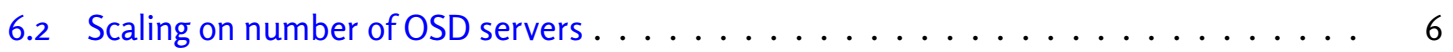

7 Ceph File System Performance: Initial Test 7

8 Improving RADOS Performance $\quad 8$

8.1 Disable Cache Mirroring on Controllers . . . . . . . . . . . . . . . . . . 9

8.2 Disable TCP autotuning . . . . . . . . . . . . . . . . . . 10

8.3 Repeating RADOS Scaling Test . . . . . . . . . . . . . . 10

9 Improving Ceph File System Performance 12

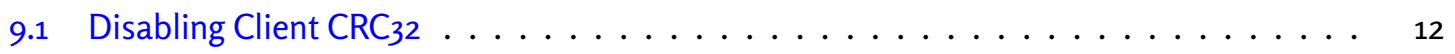

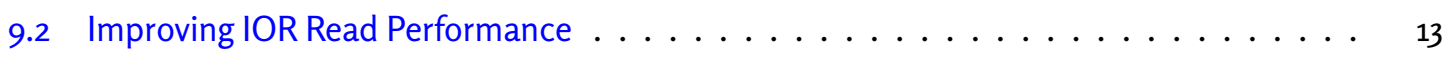

9.3 Repeating the IOR Scaling Test . . . . . . . . . . . . . . . . 14

10 Metadata Performance $\quad 15$

11 Observations and Conclusions $\quad 17$

$\begin{array}{ll}\text { Appendix A - CephFS Final Mount } & 18\end{array}$

Appendix B - OSD File System Options $\quad 18$

Appendix C - CRUSH map $\quad 19$

$\begin{array}{ll}\text { Appendix D - Final ceph.conf } & 23\end{array}$ 
Appendix E - Tuning Parameters 


\section{List of Figures}

1 DDN SFA1oK hardware and host connection diagram . . . . . . . . . . . . . 2

2 XFS read performance scaling on number of devices . . . . . . . . . . . . . . . . 4

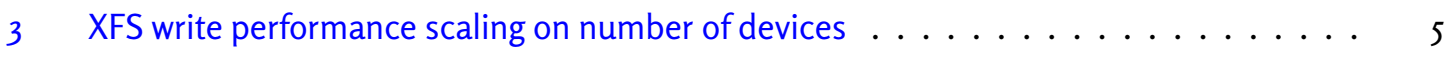

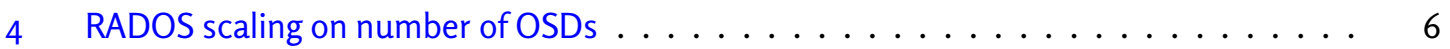

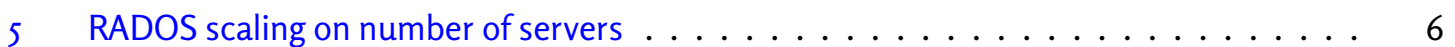

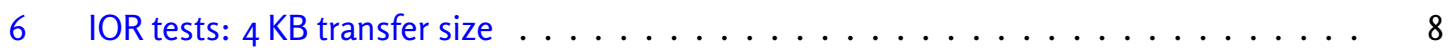

7 IOR tests: 4 MB transfer size . . . . . . . . . . . . . . . . . . 8

8 Evaluating parameter impact through sweeping test . . . . . . . . . . . . . . . 9

9 Evaluating RADOS bench after disabling cache mirroring . . . . . . . . . . . . . . 10

10 Evaluating RADOS bench after TCP auto tuning disabled . . . . . . . . . . . . . 11

11 RADOS Bench Scaling on \# of OSD, Ceph 0.64, 4 MB I/O, 8 Client Nodes . . . . . . . . . 11

12 RADOS Bench Scaling on number of servers, Ceph o.64, 4 MB I/O, 8 client nodes . . . 12

13 IOR test with disabling client-side $\mathrm{CRC}_{32} \ldots \ldots \ldots \ldots \ldots \ldots$

14 RADOS bench: Linux kernel version 3.5 vs. 3.9 . . . . . . . . . . . . . . . . . 14

15 CephFS performance with kernel changes to 3.9, IOR with 4 MB transfer size . . . . . . . 14

16 IOR Scaling Test: 4 KB and 4 MB transfer size . . . . . . . . . . . . . . . . . 15

17 File creation vs. number of clients . . . . . . . . . . . . . . . . 16

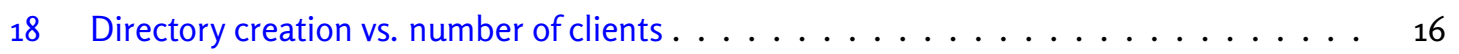

19 mdtest of file creation on Ceph $0.64 \ldots \ldots \ldots \ldots \ldots \ldots$ 


\section{Introduction}

National Center for Computational Sciences (NCCS), in collaboration with Inktank Inc, prepared this performance and scalability study of Ceph file system. Ceph originated from Sage Weil's PhD research at UC Santa Cruz around 2007 and it was designed to be a reliable, scalable fault-tolerant parallel file system. Inktank is now the major developer behind the open-source parallel file system to shepherd its development and provide commercial support.

In comparison to other parallel file systems, Ceph has a number of distinctive features:

- Ceph has an intelligent and powerful data placement mechanism, known as CRUSH. The CRUSH algorithm allows a client to pre-calculate object placement and layout while taking into consideration of failure domains and hierarchical storage tiers.

- From the start, Ceph's design anticipated managing metadata and the name space with a cluster of metadata servers. It utilized a dynamic subtree partitioning strategy to continuously adapt metadata distribution to current demands.

- Ceph's design assumes that the system is composed of unreliable components; fault-detection and fault-tolerance (e.g., replication) are the norm rather than the exception. This is in line with the expectations and future directions of Exascale computing.

- Ceph is built on top of a unified object management layer, RADOS. Both metadata and the file data can take advantage of this uniformity.

- Most of the Ceph processes reside in user-space. Generally speaking, this makes the system easier to debug and maintain. The client-side support has long been integrated into Linux mainline kernel, which eases the deployment and out-of-box experience.

As part of this effort, we set up a dedicated testbed within NCCS for the Ceph file system evaluation. The goal of our study is to investigate the feasibility of using the Ceph for our future HPC storage deployment. This report presents our experience, results, and observations. While evaluating our results, please keep in mind that Ceph is still a relatively young parallel file system and its code base is changing rapidly. In between releases, we often experienced different stability and performance outcomes. We will try to make clear in the report when such changes occurred.

This research was supported by, and used the resources of, the Oak Ridge Leadership Computing Facility, located in the National Center for Computational Sciences at ORNL, which is managed by UT Battelle, LLC for the U.S. DOE (under the contract No. DE-ACo5-0oOR22725). 


\section{Testbed Environment Description}

We used Data Direct Networks' (DDN) SFA10K as the storage backend during this evaluation. It consists of 200 SAS drives and 280 SATA drives, organized into various RAID levels by two active-active RAID controllers. The exported RAID groups by these controllers are driven by four hosts. Each host has two InfiniBand (IB) QDR connections to the storage backend. We used a single dualport Mellanox connectX IB card per host. By our calculation, this setup can saturate SFA1oK's maximum theoretical throughput $(\sim 12 \mathrm{~GB} / \mathrm{s})$. The connection diagram is illustrated in Figure 1.

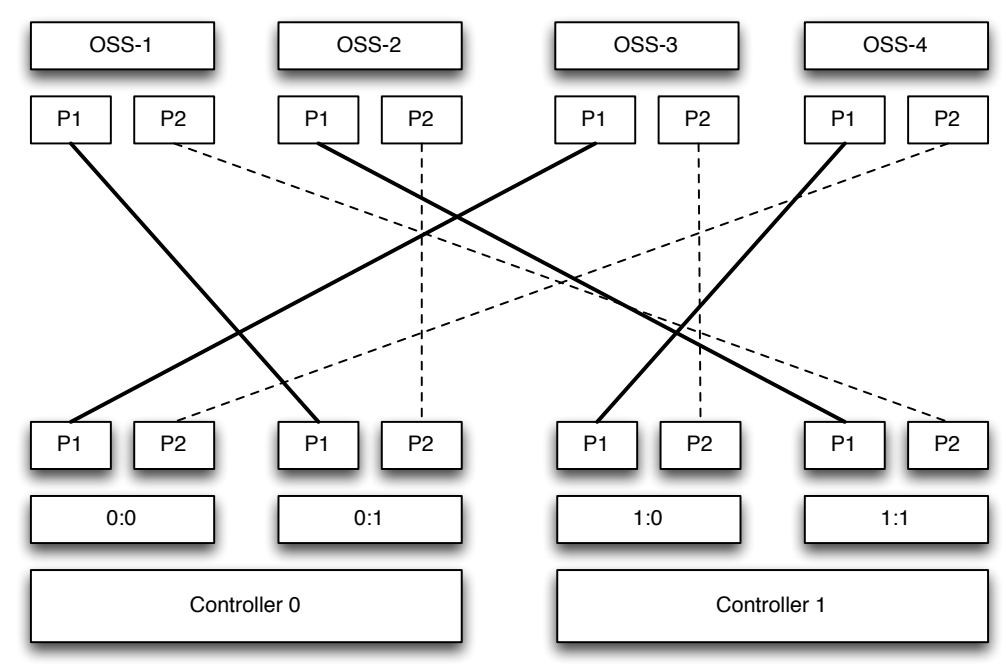

Figure 1: DDN SFA10K hardware and host connection diagram

Our Ceph testbed employs a collection of testing nodes. These nodes and their roles are summarized in Table 1. In the following discussion, we use "servers", "osd servers", "server hosts" interchangeably. We will emphasize with "client" prefix when we want to distinguish it from above.

\begin{tabular}{ll}
\hline Node & Role \\
\hline tick-mds1 & Ceph monitor node \\
spoon46 & Ceph MDS node \\
tick-oss[1-4] & Ceph OSD servers \\
spoon28-31, spoon37-41 & Ceph client nodes \\
\hline
\end{tabular}

Table 1: Support nodes involved in Ceph testbed

All hosts (client and servers) were configured with Redhat 6.3 and kernel version 3.5.1 initially, and later upgraded to 3.9 (rhl-ceph image), Glibc 2.12 with syncfs support, locally patched. We used the Ceph 0.48 and 0.55 release in the initial tests, upgraded to 0.64 and then to $0.67 \mathrm{RC}$ for a final round of tests. 


\section{Baseline Performance}

\subsection{Block I/O over Native IB}

We first established baseline performance by measuring block $1 / 0$ performance. At the block-level, with each LUN configured as a RAID6 8+2 array, we had the following results as shown in Table 2.

\begin{tabular}{ll}
\hline SAS single LUN sequential read & $1.4 \mathrm{~GB} / \mathrm{s}$ \\
SATA single LUN sequential read & $955 \mathrm{MB} / \mathrm{s}$ \\
Single host with four SAS LUNs & $2.8 \mathrm{~GB} / \mathrm{s}$ \\
Single host with seven SATA LUNs & $2.6 \mathrm{~GB} / \mathrm{s}$ \\
\hline
\end{tabular}

Table 2: Block I/O performances on single LUN and single host

Single host in this table refers to one of four tick-oss nodes. Four tick-oss nodes drive the SFA1oK backend storage. Overall, we observe that the entire system can perform at $11 \mathrm{~GB} / \mathrm{s}$, compared to DDN SFA10K's theoretical maximum of $12 \mathrm{~GB} / \mathrm{s}$.

\subsection{IP over IB}

Ceph uses the BSD Sockets interface in SOCK_STREAM mode (i.e., TCP). Because our entire testbed is IBswitched, we used IP over IB (IPoIB) for networking ${ }^{1}$. Through simple netperf tests, we observed that a pair of hosts connected by IB QDR using IPoIB can transfer data at $2.7 \mathrm{~GB} / \mathrm{s}$ (the hosts are tuned per recommendations from Mellanox). With all four hosts (OSD servers), we expect the aggregate throughput to be in the neighborhood of $10 \mathrm{~GB} / \mathrm{s}$.

Unfortunately there was not enough time to do more detailed analysis of the network performance. However, as we observed later, RADOS is performing no more than $8 \mathrm{~GB} / \mathrm{s}$ driven by four server hosts. This confirms that we have provisioned enough network bandwidth. In other words, IP over IB is not a bottleneck in this case.

\section{System Tuning}

Base upon past experience and further experimentation, we started out with the following set of tuning parameters on OSD servers:

\footnotetext{
${ }^{1}$ As of writing of this report, Inktank is investigating using rsockets to improve performance with IB fabric
} 


\begin{tabular}{ll}
\hline nr_requests & 2048 \\
read_ahead_kb & 4096 \\
scheduler & deadline \\
\hline
\end{tabular}

Table 3: System tuning parameters

\section{XFS Performance As Backend File System}

Ceph supports multiple backend file systems including Btrfs, Ext4, and XFS. Due to its maturity and Inktank's recommendations, we chose XFS. ${ }^{2}$ We experimented with XFS to acquire a set of configuration parameters which provided optimal performance for the SFA1OK. We sampled a selected set of parameters (block size, queue depth, request size, sequential read and write). We settled on the following configuration: mount with nobarrier, noat im, inode64 options. The inode64 option had a notable improvement on sequential write (around 20\%).

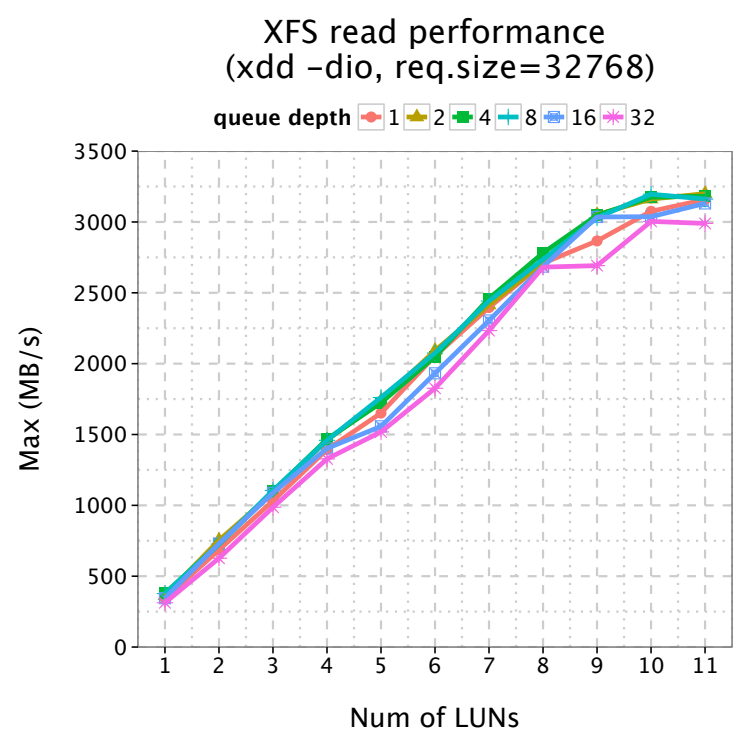

Figure 2: XFS read performance scaling on number of devices

The read and write performance results are summarized in Figures 2 and 3, respectively. These graphs show scaling behavior over the number of LUNs, which indicates that XFS can reach the peak write performance with just five SATA LUNs. Increasing number of LUNs beyond 5, degradation happened. Also, we did not observe any obvious differences in performance when varying the queue depth parameter. For these tests, we used the xdd benchmark with direct l/O and a request size of $32 \mathrm{~KB}$.

${ }^{2}$ It should be noted that in testing cases while we can compare XFS vs. Brtfs, Brtfs generally shows better performance results. 


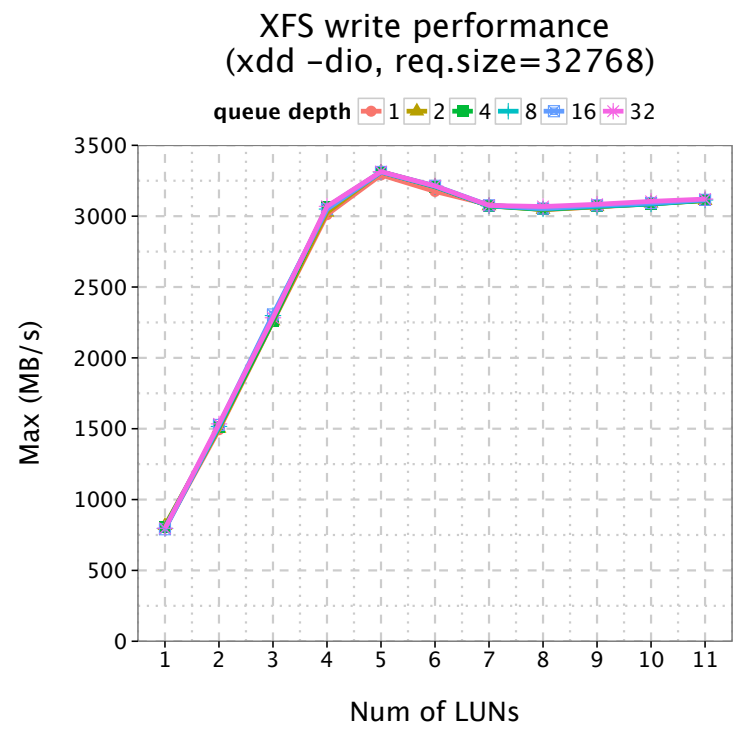

Figure 3: XFS write performance scaling on number of devices

\section{Ceph RADOS Scaling: Initial Test}

RADOS is a reliable distributed object store, the foundational component for CephFS file system. There are two types of scaling tests we are interested at the RADOS layer:

- scaling on the number of OSD servers

- scaling on the number of OSDs per OSD server

Our system setup poses some limitations on the scalability tests we wanted to perform. In particular, we currently had four OSD servers, eight clients, and eleven OSD servers per client. The scaling tests, therefore, will be within these constraints.

We used the open-source RADOS Bench tool, developed by Inktank, to perform our initial performance analysis of the underlying RADOS layer. RADOS Bench simply writes out objects to the underlying object storage as fast as possible, and then later reads those objects in the same order as they were written.

We observed that using two or more client processes and many concurrent operations are important when performing these tests. We tested eight client processes with 32 concurrent 4 MB objects in flight each. We created a pool for each RADOS Bench process to ensure that object reads come from independent pools (RADOS Bench is not smart enough to ensure that objects are not read by multiple processes and thus possibly cached). A sync and flush is performed on every node before every test to 
ensure no data or metadata is in cache. All tests were run with replication set to one. The backend file systems were XFS, BTRFS and EXT4 file systems were not tested at this time.

\subsection{Scaling on number of OSDs per server}

In the following test, a single Ceph host drives $n$ OSDs, where $n$ increases from one to eleven. The result is illustrated in Figure 4. We ran the test against a single client with four concurrent processes. In this test case, we observe that the OSD server exhibits near linear scalability up to nine OSDs, and is still trending upwards at eleven OSDs. This suggests that we have not reached the saturation point yet. Additional testing would require provisioning more OSDs on the SFA10K backend.

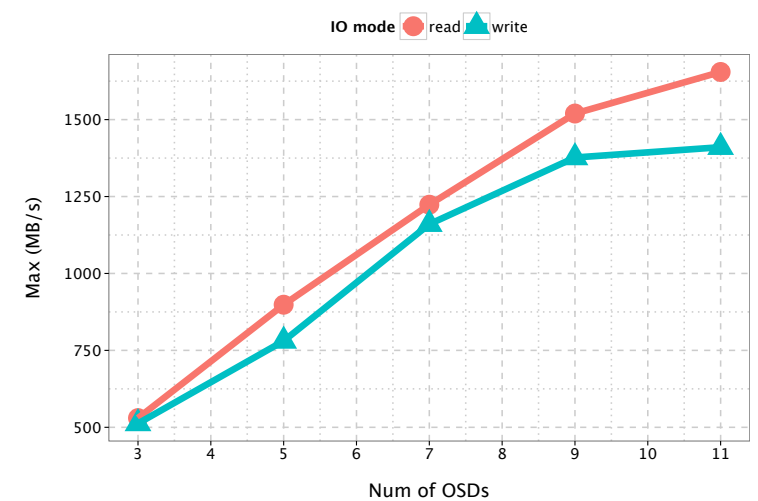

Figure 4: RADOS scaling on number of OSDs

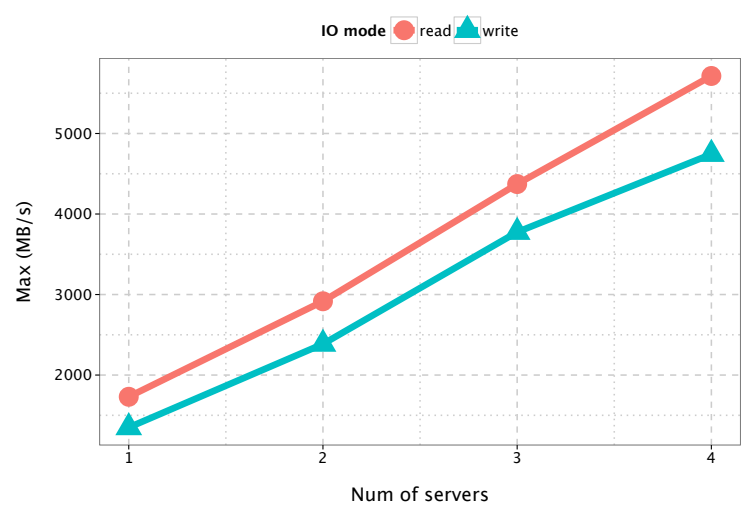

Figure 5: RADOS scaling on number of servers

\subsection{Scaling on number of OSD servers}

In this test, we exercise OSD servers from one to four, driven by four hosts each with four RADOS Bench process. Each additional OSD server adds eleven more OSDs into play. We observe that Ceph exhibits linear scaling with regard to number of servers as well, at least in the given set of servers. However, the peak performance we are seeing is about the half of what are expecting from the SFA10K (compare to the baseline block I/O performance number presented in Section 3).

For writes, the lost performance is attributed to the way Ceph performs journaling: Ceph does not support meta-data only journaling, therefore every write is the equivalent of a double-write: once to the data device, once to the journaling device. This effectively cuts the observed system bandwidth in half. That said, it does not explain the read performance - it is a little better than write, but still far from the theoretical maximum. 


\begin{tabular}{l|l}
\hline IOR parameter & Note \\
\hline$-F$ & file per process \\
\hline- a POSIX & use POSIX API \\
\hline$-w-r-C$ & $\begin{array}{l}\text { do both write and read test, }-C \text { is to change task or- } \\
\text { cering for read back so it will not read from the write } \\
\text { cache. }\end{array}$ \\
\hline- i $3-$ d 5 & 3 iterations and delay 5 seconds betewen iterations \\
\hline$-e$ & perform fsync() upon POSIX write close \\
\hline- b $8 g$ or $16 \mathrm{~g}$ & the block size \\
\hline- t $4 \mathrm{k}$ to $4 \mathrm{~m}$ & the transfer size \\
\hline-0 file & mandatory test file \\
\hline
\end{tabular}

Table 4: IOR parameter setup

\section{Ceph File System Performance: Initial Test}

We used the synthetic IOR benchmark suite for file system level performance and scalability test. The particular parameter setup is show in Table 4. Each client node has 6 GB of physical memory, the block size is set so as to mitigate cache effects. In addition, the test harness program issues the following commands at the beginning of each test:

\# sync

\# echo 3 | tee /proc/sys/vm/drop_caches

Here, $o$ is the default value of drop_caches; 1 is to free pagecaches, 2 is to free dentries and inodes, 3 is to free pagecache, dentries, and inodes.

Our first round of tests was less than ideal as we encountered various issues. For the sake of completeness, we first summarize the results, then discuss further tuning efforts and improvements.

The full permutation of IOR parameters were not explored due to I/O errors we encountered. We were, however, able to record results in two extreme cases as far as transfer size is concerned: $4 \mathrm{~KB}$ and $4 \mathrm{MB}$, using a fixed number of OSD servers (4) and fixed block size ( $8 \mathrm{~GB})$, the results are illustrated in Figure 6 and 7, we make the following observations: 


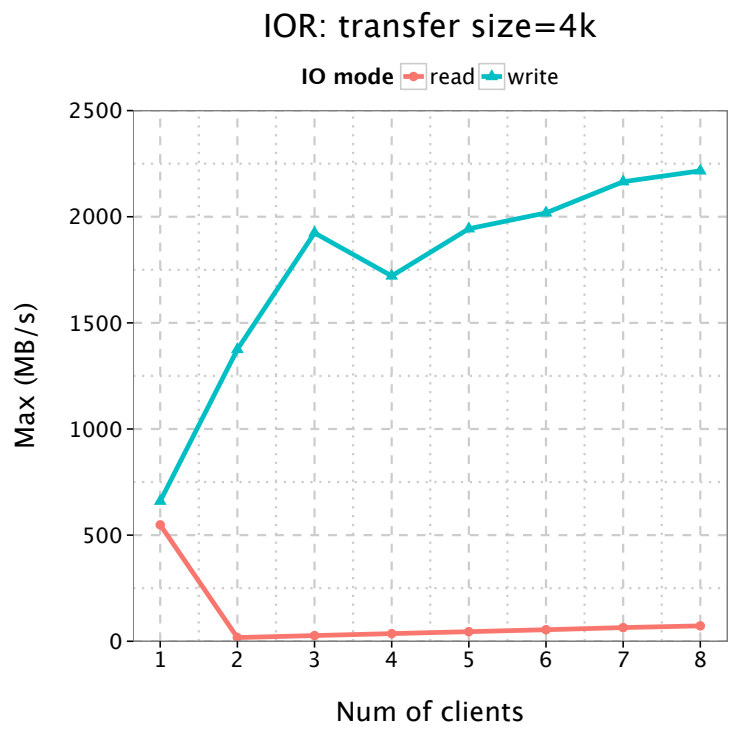

Figure 6: IOR tests: 4 KB transfer size

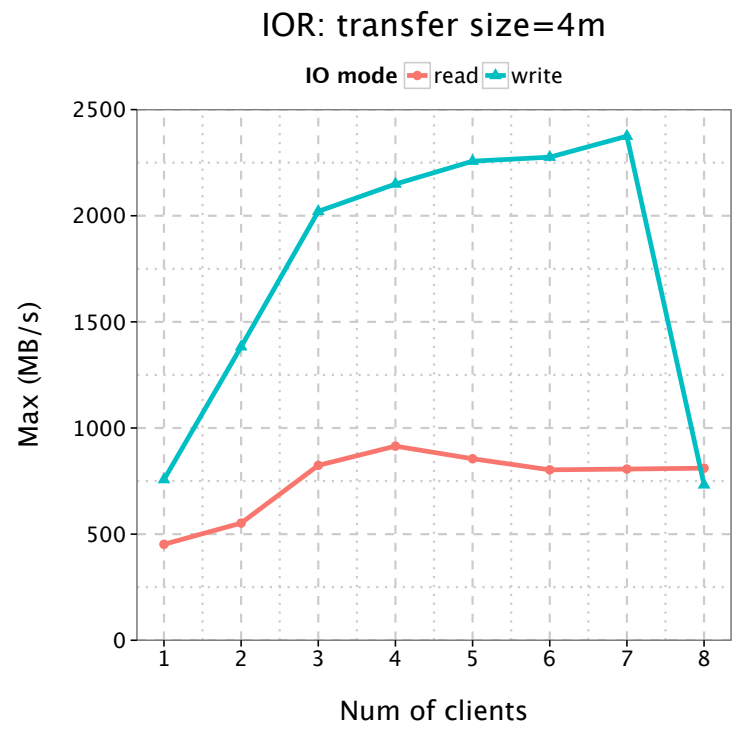

Figure 7: IOR tests: 4 MB transfer size

- The small read (4 KB transfer size) performance is almost an anomaly - we will investigate why it is so low compare to write performance and present improved results in Section 9.

- The large read (4 MB transfer size) performance is almost half of the RADOS read performance.

- The write performance is also about half of what we can obtain from RADOS Bench. When number of clients reaches 8 , there is a significant performance drop as well.

We will describe the efforts and results on performance improvement in the following sections.

\section{Improving RADOS Performance}

After the initial test results, we tried various combinations of tweaks including changing the number of filestore op threads, putting all of the journals on the same disks as the data, doubling the OSD count, and upgrading Ceph to a development version which reduces the seek overhead caused by pginfo and pglog updates on XFS (these enhancements are now included as of the Ceph Cuttlefish release, vo.61). The two biggest improvements resulted from disabling $\mathrm{CRC}_{32 \mathrm{C}}$ checksums and increasing the OSD count on the server. With these changes, we are seeing better results.

We ran a script written by Inktank for internal Ceph testing to perform sweeps over Ceph configuration parameters to examine how different tuning options affect performance on the DDN platform. The 


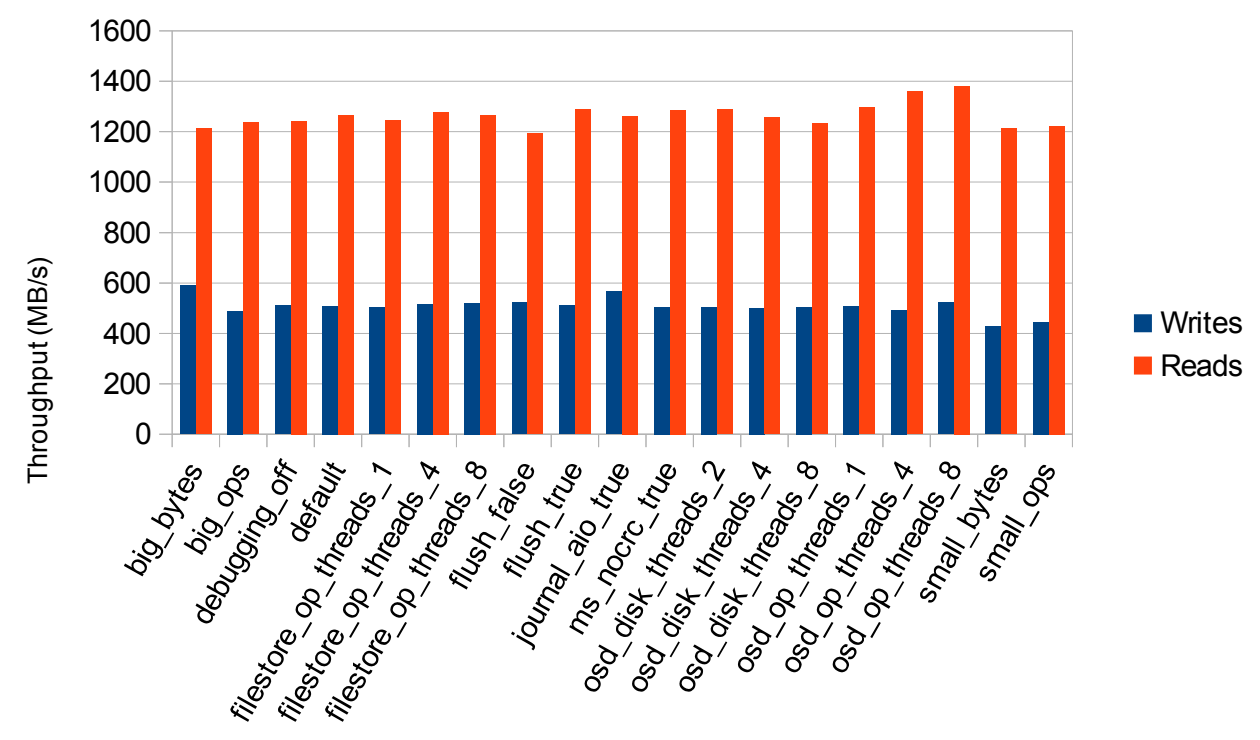

Figure 8: Evaluating parameter impact through sweeping test

result of this parameter probing is illustrated in Figure 8. Please refer to Appendix E for explanations of these probed parameters.

As a result of this testing, we improved performance slightly by increasing the size of various Ceph buffers and queues, enabling AIO journals, and increasing the number of OSD op threads.

\subsection{Disable Cache Mirroring on Controllers}

During a second round of test performed by Inktank, we noticed a dramatic drop on RADOS performance: even though write throughput on individual server met the expectation, it did not scale across servers.

We spent a significant amount of time investigating this phenomenon. Ultimately, we were able to replicate this finding when running concurrent disk throughput tests directly on the servers without Ceph involved. The second RAID processor on each DDN controller would max out when three or more LUNs were written concurrently. It turns out the root of the problem was a regression on DDN firmware update - in particular, the cache mirroring was not behaving as it should. ${ }^{3}$

With cache mirroring disabled, write performance when using all four servers improved dramatically, as illustrated in Figure 9. With BTRFS, for example, we hit over $5.5 \mathrm{~GB} / \mathrm{s}$ from the clients. When accounting for journal writes, that is over $11 \mathrm{~GB} / \mathrm{s}$ to the disks and very close to what the DDN chassis is capable

\footnotetext{
${ }^{3} \mathrm{DDN}$ recently released a new firmware version and we were told the issue has been fixed. Unfortunately, we didn't get a chance to verify it during our test cycle.
} 


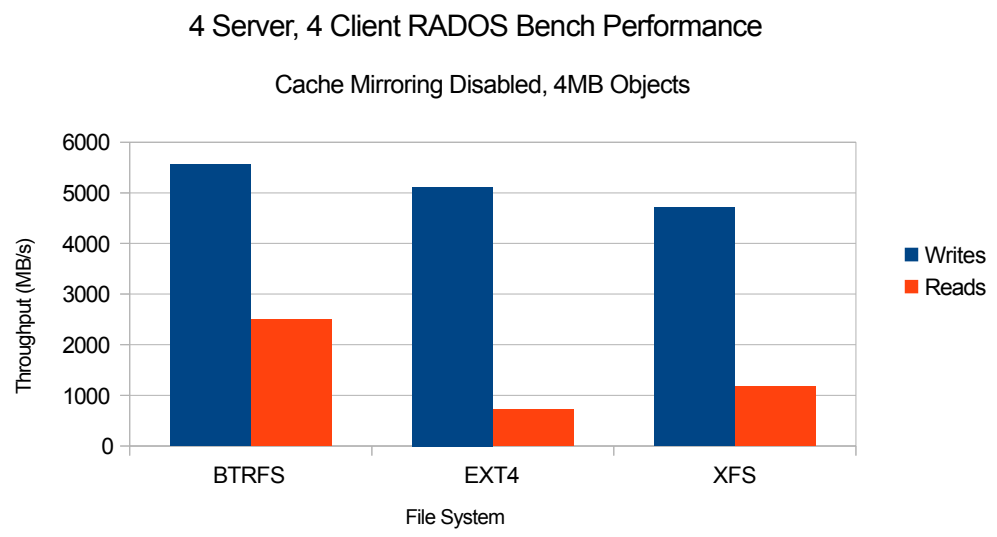

Figure 9: Evaluating RADOS bench after disabling cache mirroring

of doing. Unfortunately, read performance did not scale as well.

\subsection{Disable TCP autotuning}

During these tests, a trend that previously had been seen became more apparent. During reads, there were periods of high performance followed by periods of low performance or outright stalls that could last for up to 20 seconds at a time. After several hours of diagnostics, Inktank observed that outstanding operations on the clients were not being shown as outstanding on the OSDs. This appeared to be very similar to a problem Jim Schutt at Sandia National labs encountered with TCP autotuning in the Linux kernel. ${ }^{4}$ TCP auto tuning enables TCP window scaling by default and automatically adjusts the TCP receive window for each connection based on link conditions such as bandwidth delay product. We have observed this will make a notable improvement on Ceph read performance, as the results shown in Figure 10.

Luckily, the fix was fairly straight forward by issuing the following command on all nodes:

echo $0 \mid$ sudo tee /proc/sys/net/ipv4/tcp_moderate_rcvbuf

Recent versions of Ceph work around this issue by manually controlling the TCP buffer size. The testing at ORNL directly influenced and motivated the creation of this feature!

\subsection{Repeating RADOS Scaling Test}

We now repeated the previous RADOS scaling tests with these improvements in place. The first test was done on a single node with RADOS Bench to see how close the underlying object store could get to the

4http: //marc. info/?l=ceph-devel\&m=133009796706284\&w=2 
4 Server, 4 Client RADOS Bench Performance

Cache Mirroring Disabled, TCP Autotuning Disabled, 4MB Objects

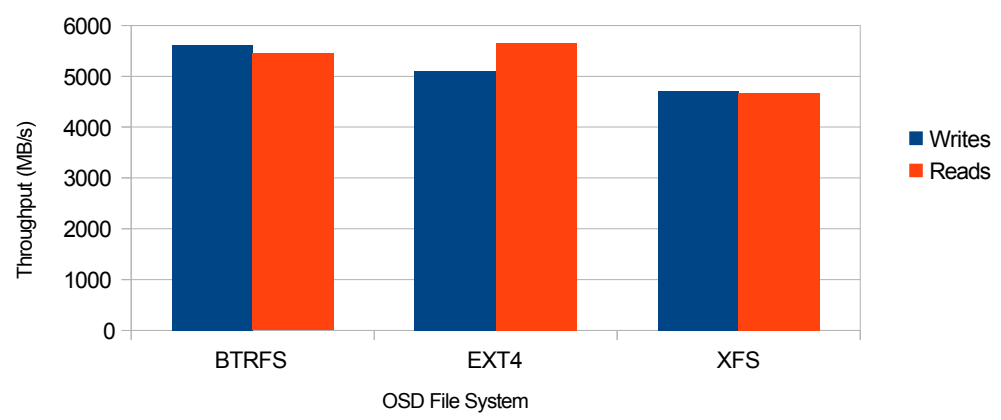

Figure 10: Evaluating RADOS bench after TCP auto tuning disabled

node hardware limitations as the number of OSDs/LUNs used on the node increased. Note all the tests performed were against XFS-formatted storage.

4MB I/Os, 8 Client Nodes

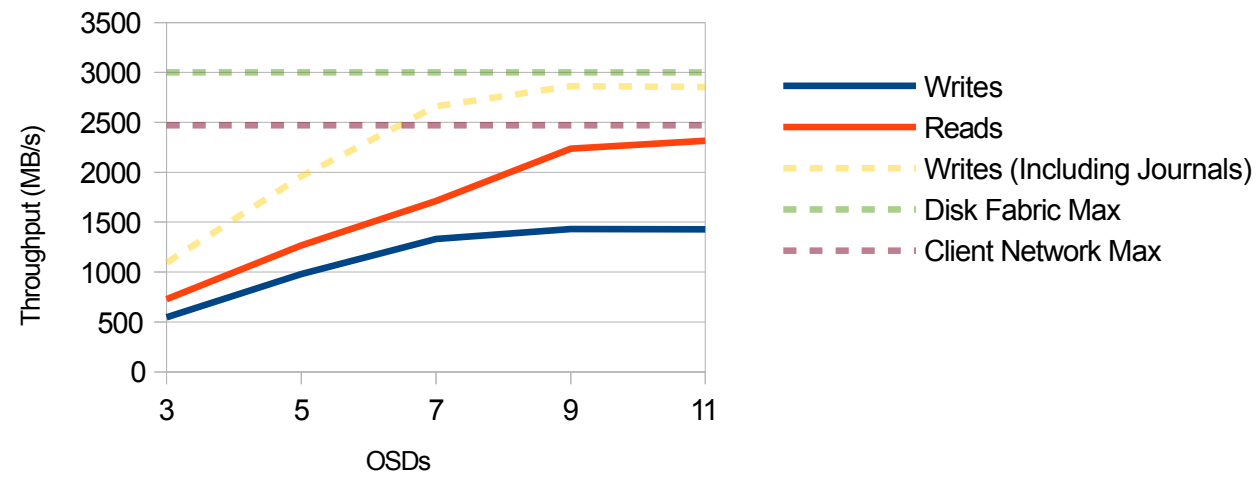

Figure 11: RADOS Bench Scaling on \# of OSD, Ceph o.64, 4 MB I/O, 8 Client Nodes

In the single server case as shown in Figure 11, "Writes (including Journals)" refers to how much data is actually being written out the DDN chasis, and blue line is how much data the clients are writing. We observe that performance gets very close to the hardware limits at roughly 9 OSDs per server and then mostly levels out.

We also repeated tests looking at RADOS Bench performance as the number of OSD server nodes increases from one to four. The results are summarized in Figure 12. As the number of nodes increases, performance scales nearly linearly for both reads and writes. 
4MB I/Os, 8 Client Nodes

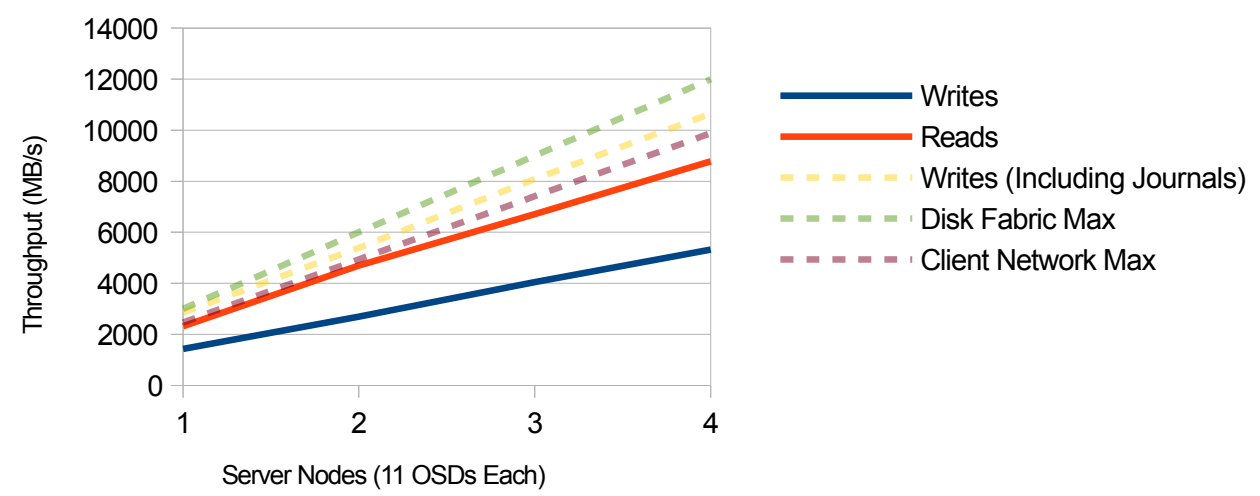

Figure 12: RADOS Bench Scaling on number of servers, Ceph o.64, 4 MB I/O, 8 client nodes

\section{Improving Ceph File System Performance}

The initial stability issues mentioned in Section 7 are fixed by migrating from Ceph version 0.48/0.55 to 0.64 , the latest stable version at the time of writing this report. Upgrading to the latest stable Ceph release allowed us to run a full IOR parameter sweep for the first time since we started evaluating the performance and scalability of the Ceph file system. This is another sign of how much Ceph development is currently in flux.

Another fix introduced by Ceph version 0.64 was in pool creation. The default data pool used by previous Ceph version were set to $2 x$ replication by mistake. This potentially halved the write performance. With version 0.64 we explicitly set the replication level to 1 , which is the preferred value for a HPC environment like ours running on high-end and reliable storage backend hardware (e.g. DDN SFA1oK).

Even with these two changes in place, less-than-ideal write performance and very poor read performances were observed during our tests. We also observed that by increasing the number of IOR processes per client node, the read performance degraded even further indicating some kind of contention either on the clients or on the OSD servers.

\subsection{Disabling Client $\mathrm{CRC}_{32}$}

At this point, we were able to both make more client nodes available for Ceph file system-level testing and also install a profiling tool called perf that is extremely useful for profiling both kernel and user space codes. Profiling with perf showed high CPU utilization on test clients due to crc32c processing in the Ceph kernel client. crc32 checksums can be disabled by changing the CephFS mount options:

mount -t ceph 10.37.248.43:6789:/ /mnt/ceph -o name=admin, nocrc 
With client $\mathrm{CRC}_{32}$ disabled, we repeated the IOR tests. New results are shown in in Figure 13.

No Client CRC32, 1 Process per Client, 4 Server Nodes

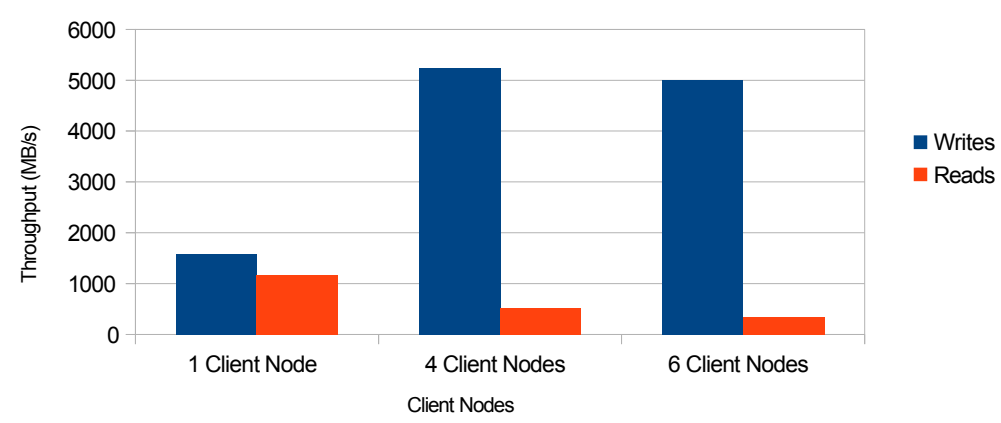

Figure 13: IOR test with disabling client-side $\mathrm{CRC}_{32}$

We observed that IOR write throughput increased dramatically and is now very close and comparable to the RADOS Bench performance. Read performance continued to be poor and continued to scale inversely with the increasing number of client processes. Please note that, since these tests were performed, Inktank has implemented SSE4-based $\mathrm{CRC}_{32}$ code for Intel CPUs. While any kernel based $\mathrm{CRC}_{32}$ processing should have already been using $\mathrm{SSE}_{4}$ instructions on Intel CPUs, this update will allow any user-land Ceph processes to process $\mathrm{CRC}_{32}$ checksums with significantly less overhead.

\subsection{Improving IOR Read Performance}

Deeper analysis with perf showed that there was heavy lock contention during parallel compaction in the Linux kernel memory manager. This behavior was first observed roughly in the kernel 3.5 time frame which was the kernel installed on our test systems. 5

We upgraded our test systems with kernel version 3.9 and performed RADOS Bench test. The results were extremely positive and presented in Figure 14.

As can be seen, with the 3.9 kernel, while there was a slight improvement on write performance, read performance improved dramatically. In addition to the kernel change, Sage Weil from InkTank suggested increasing the amount of CephFS client kernel read-ahead cache size as:

mount -t ceph 10.37.248.43:6789:/ /mnt/ceph -o

name $=$ admin, nocrc, readdir_max_bytes $=4104304$, readdir_max_entries $=8192$

IOR results reflecting the read-ahead cache size change are presented in Figure 15.

By installing a newer kernel, increasing read-ahead cache size, and increasing the number of client IOR processes, we were able to achieve very satisfactory I/O performance.

\footnotetext{
5For more information, please refer to http://lwn. net/Articles/517082/ and https://patchwork.kernel.org/patch/ $1338691 \%$.
} 


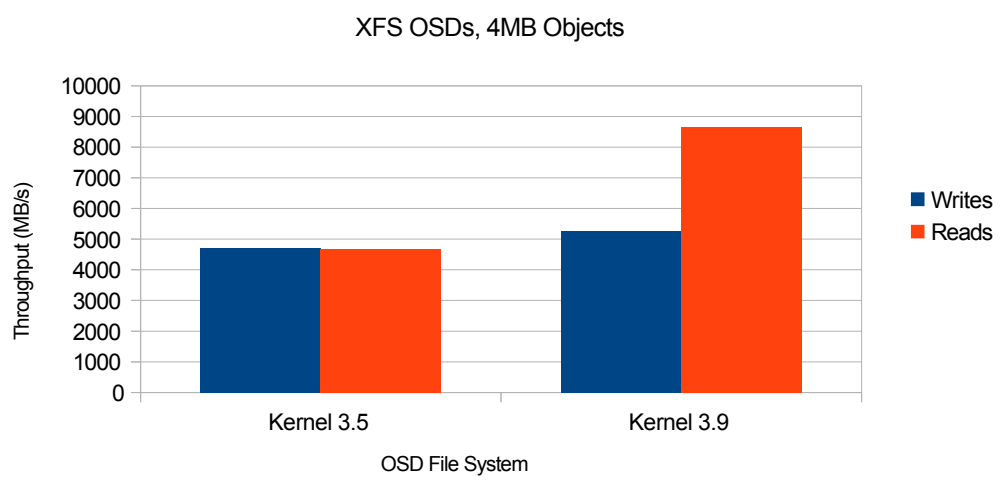

Figure 14: RADOS bench: Linux kernel version 3.5 vs. 3.9

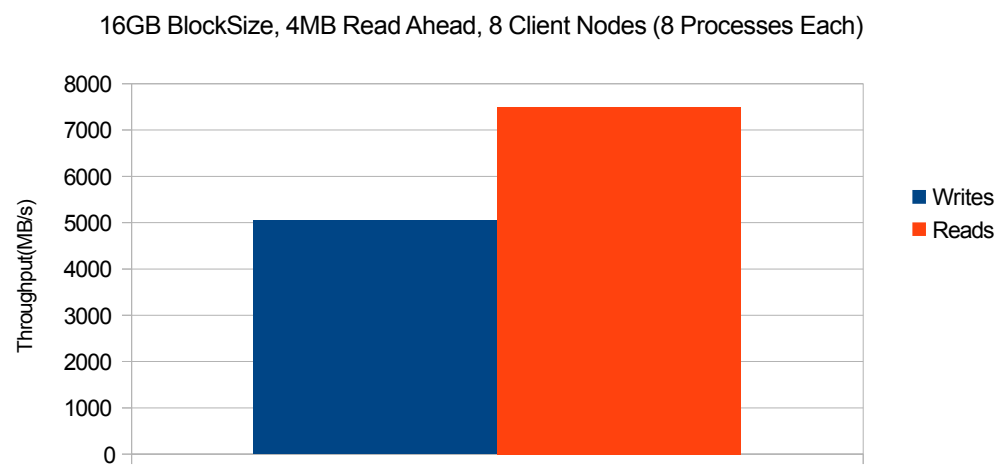

Figure 15: CephFS performance with kernel changes to 3.9, IOR with 4 MB transfer size

\subsection{Repeating the IOR Scaling Test}

As before, we ran IOR scaling tests with two cases: transfer size $4 \mathrm{~KB}$ and $4 \mathrm{MB}$. These results are illustrated in Figure 16. As expected, we saw saw improved read and write performance. These new read and write performance are in line with observed RADOS bench performance.

Throughout our IOR testing, we observed that the average write throughput is lower than the maximum. This behavior was observed during other tests as well, indicating that we may have periods of time where write throughput is temporarily degrading. Despite these issues, performance generally seems to be improving with respect to increasing number of clients. Writes seem to be topping out at around 5.2 $\mathrm{GB} / \mathrm{s}$ (which is roughly what we would expect). Reads seem to be topping out anywhere from 5.6-7 GB/s, however it is unclear if read performance would continue scaling with more clients and get closer to 8 $\mathrm{GB} / \mathrm{s}$ we observed with RADOS Bench. 


\subsection{XFS 4K IOR Throughput}

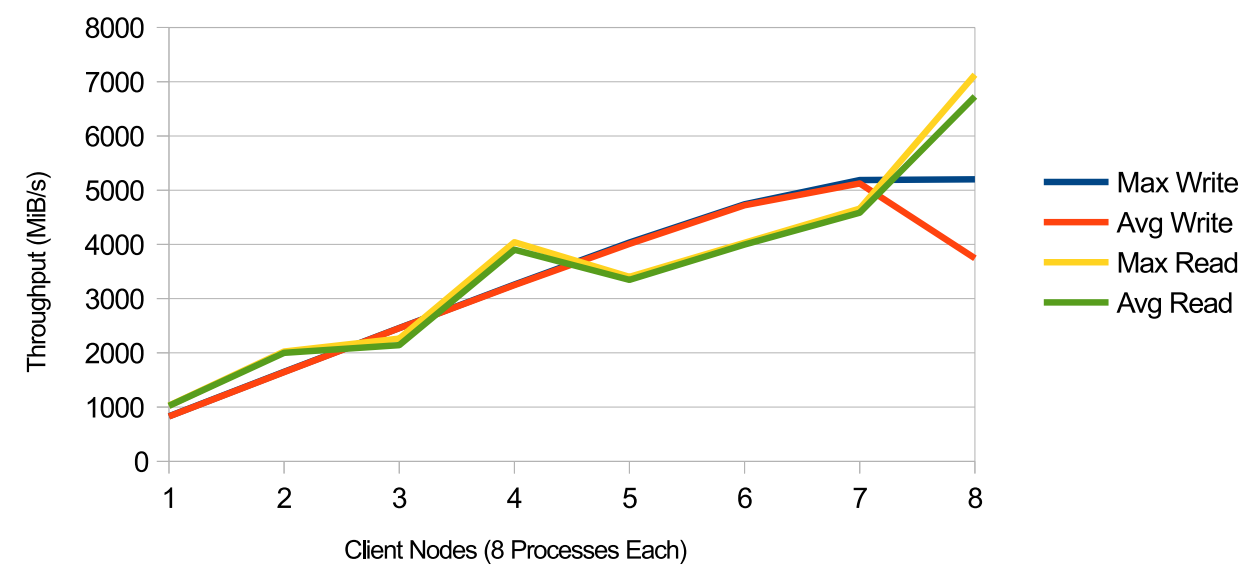

\subsection{XFS 4M IOR Throughput}

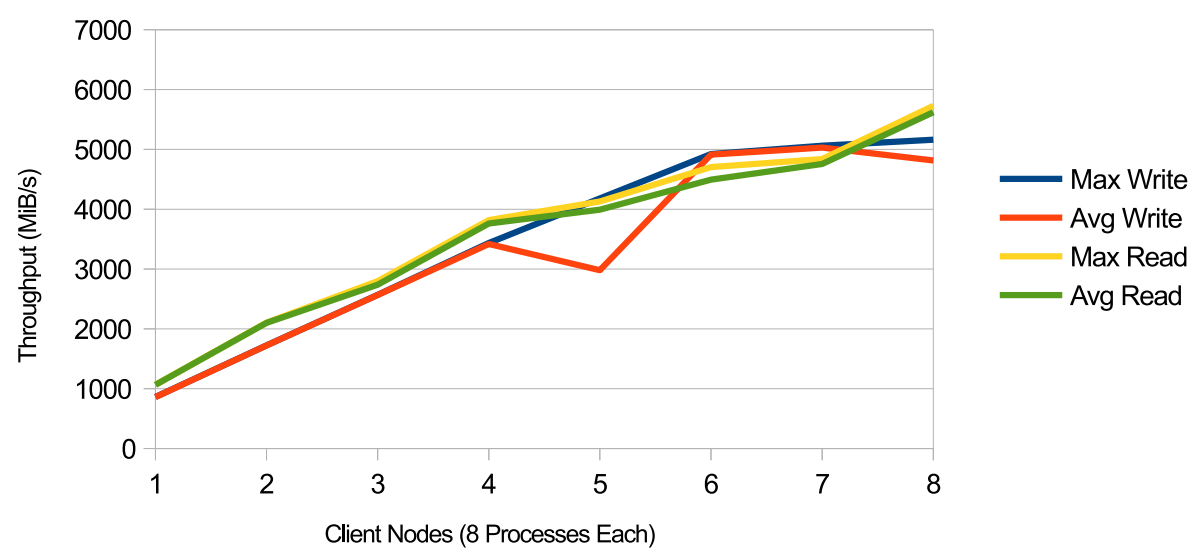

Figure 16: IOR Scaling Test: 4 KB and 4 MB transfer size

\section{Metadata Performance}

In our particular setup, we only had one metadata server (MDS) configured. Therefore, this is not a scalability test on the performance of Ceph clustered MDS, which would have been very interesting. Instead, we focus on a single MDS performance and exercise it with up to 8 clients to observe the single MDS performance scaling. We used mdtest as our benchmark tool. Mdtest parameters used for this test are: 


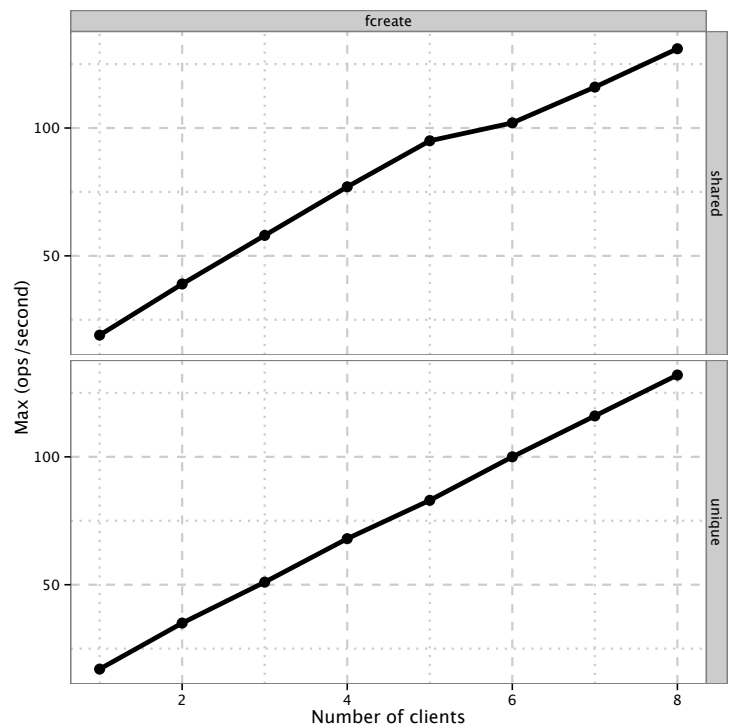

Figure 17: File creation vs. number of clients

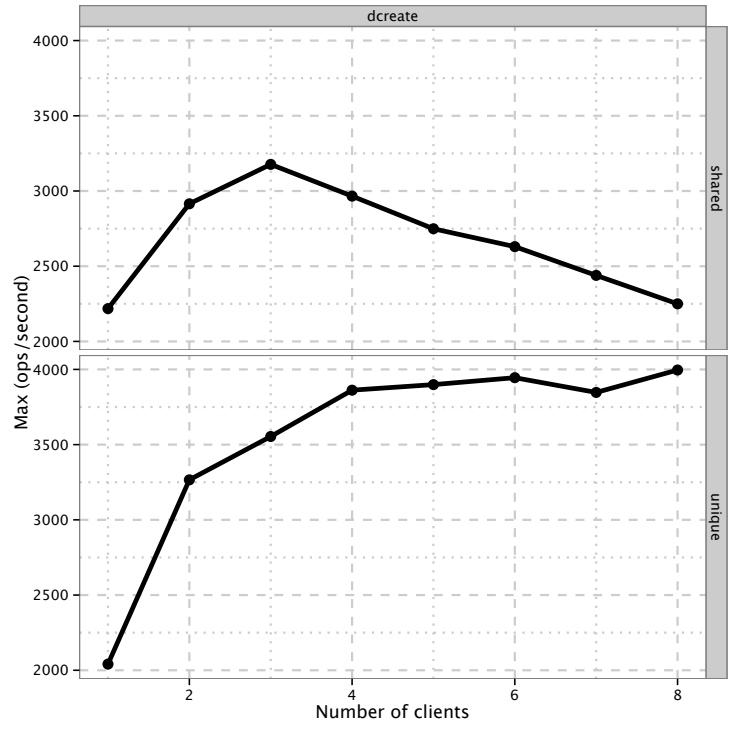

Figure 18: Directory creation vs. number of clients

- -w 1048576 -y: for each created file, we write 1 MB data and perform a sync operation. This is a more realistic use case scenario than just open, close and removal sequence of metadata operations.

- $-n$ 1000: Per client file and directory workload. For eight clients, the total number of files and directories in the workload is 8,000 . Since we did not specify either $-D$ or $-F$, this is a mixture of both.

- -d /mnt/cephfs/tmp: we do specify a directory, but unlike under Lustre file system, where you can have single client multiple mounts (for increasing workload per client), here we just give the test an explicit home.

- $-\mathrm{u}$ : without this option, we are exercising shared directory; with this option, we are exercising unique directories.

Each test iterates five times and we are presenting the maximum out of all five iterations. We summarize the results as follows:

- With either shared or unique directory (-u), stat operations for directories and files exhibit strong linear scaling. Same strong linear scaling is also observed for file read operations.

- While the other operations seems unaffected or flat by the number of clients, it is not so if we zoom 
in, see 17 and 18: as number of clients increases, we observed the contention for shared directory. The performance degradation amount to $50 \%$ or more.

- Though the same saturation (or degradation) trend was not observed for file creation operation, it is likely due to lack of workload stress on MDS.

The results also show that file creation rate is significantly lower than directory creation rate. We stipulated two contributing factors: one is the file creation is associated with 1 MB data write and followed by a fsync operation, which is a rather heavy weight operation compare to directory creation. Another factor is that we obtained above results from early version of Ceph without all the tunings and improvement applied. Therefore, we repeated the same test on the latest CephFS 0.64. The result is illustrated in Figure 19. As expected, we observed a significant improvement on the file creation rate. We also note that as number of clients increase, the file creation rate decreases rapidly, which suggested some form of lock contention. This presents some interesting issues to be investigated further.

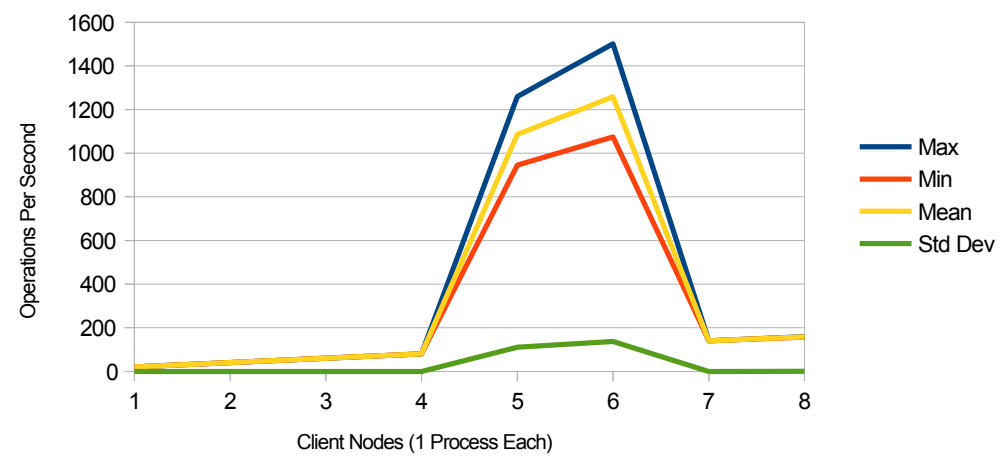

Figure 19: mdtest of file creation on Ceph 0.64

\section{Observations and Conclusions}

Below are our preliminary observations from mostly performance perspective:

- Ceph is built on the assumption that the underlying hardware components are unreliable, with little or no redundancy and failure detection capability. This assumption is not valid for high-end HPC centers like ours. We have disabled replication for pools, we haven't measured and quantified processing overhead and we do not know yet if this would be significant.

- Ceph performs metadata + data journaling, which is fine for host systems that has locally attached disk. However, this presents a problem in DDN SFA1oK-like hardware, where the backend LUNs 
are exposed as block devices through SCSI Request Protocol (SRP) over IB. The journaling write requires twice the bandwidth compare to Lustre-like metadata-only journaling mechanism. For Ceph to be viable in this facility, journaling operations will need to further design and engineering.

- We observed consistent results and linear scalability at the RADOS level. However, we did not observe the same at the file system level. We have experienced large performance swings during different runs, very low read performance when transfer size is small, and $\mathrm{l} / \mathrm{O}$ errors tend to happen when system is under stress (more clients and large transfer sizes). These are not particularly reproducible results, but it suggests that there are opportunities for code improvement and maturation.

\section{Appendix A - CephFS Final Mount Command}

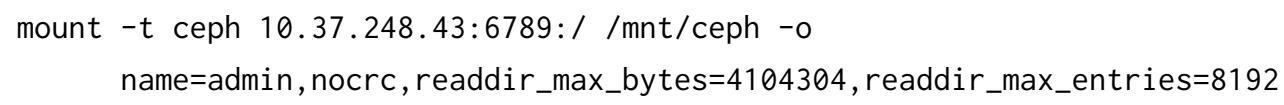

\section{Appendix B - OSD File System Options}

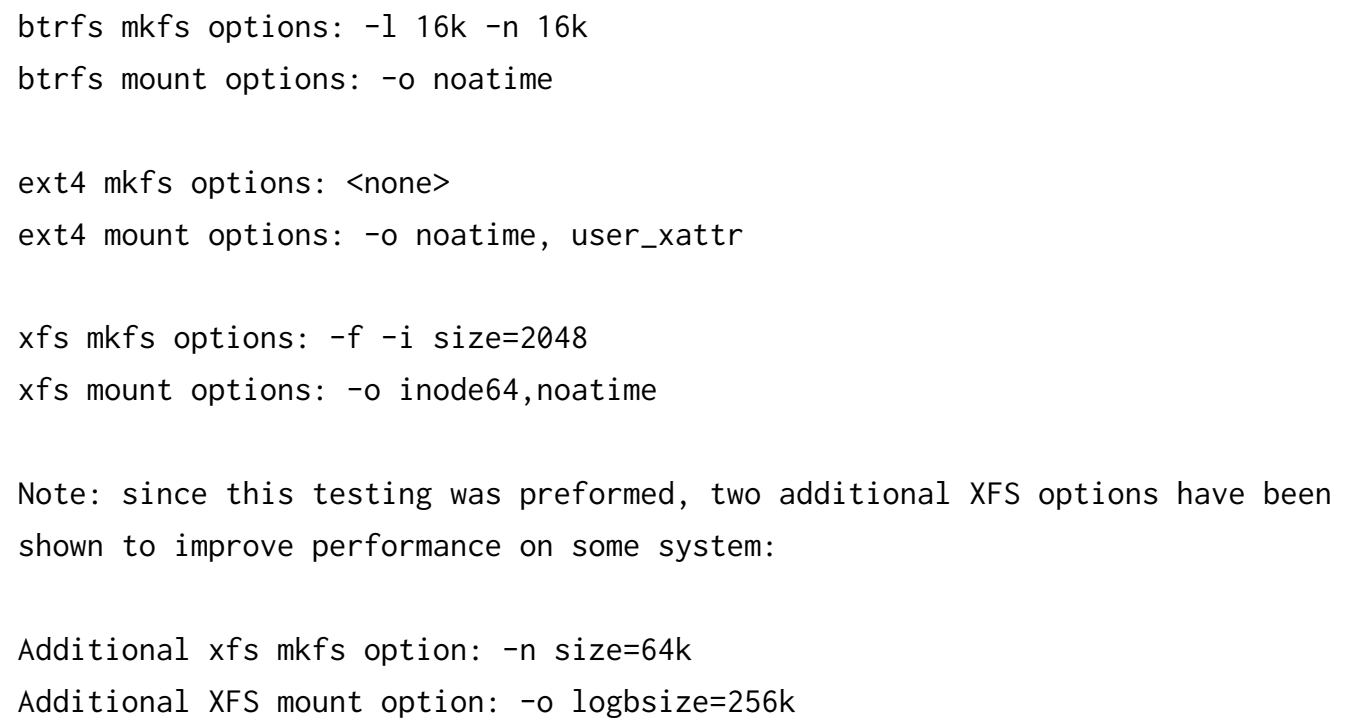




\section{Appendix C - CRUSH Map}

This crush map is just an example of full OSD mapping. As we create and re-create different testbed setups with different OSD configurations, the map will change accordingly.

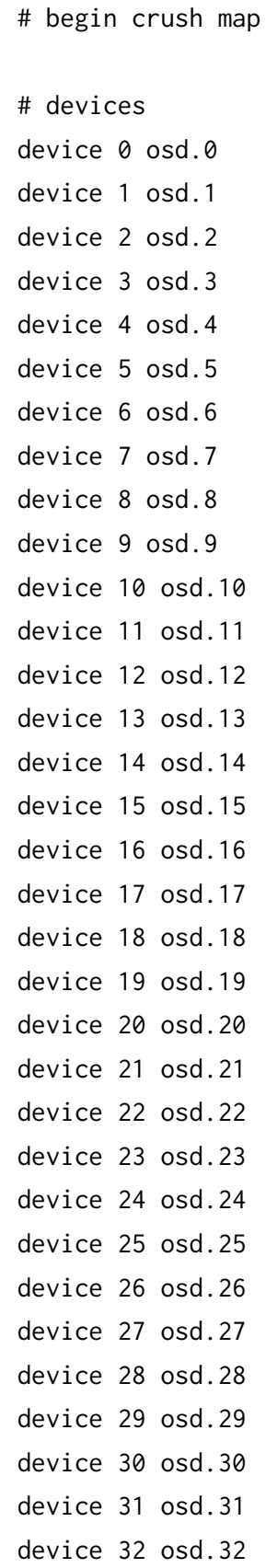




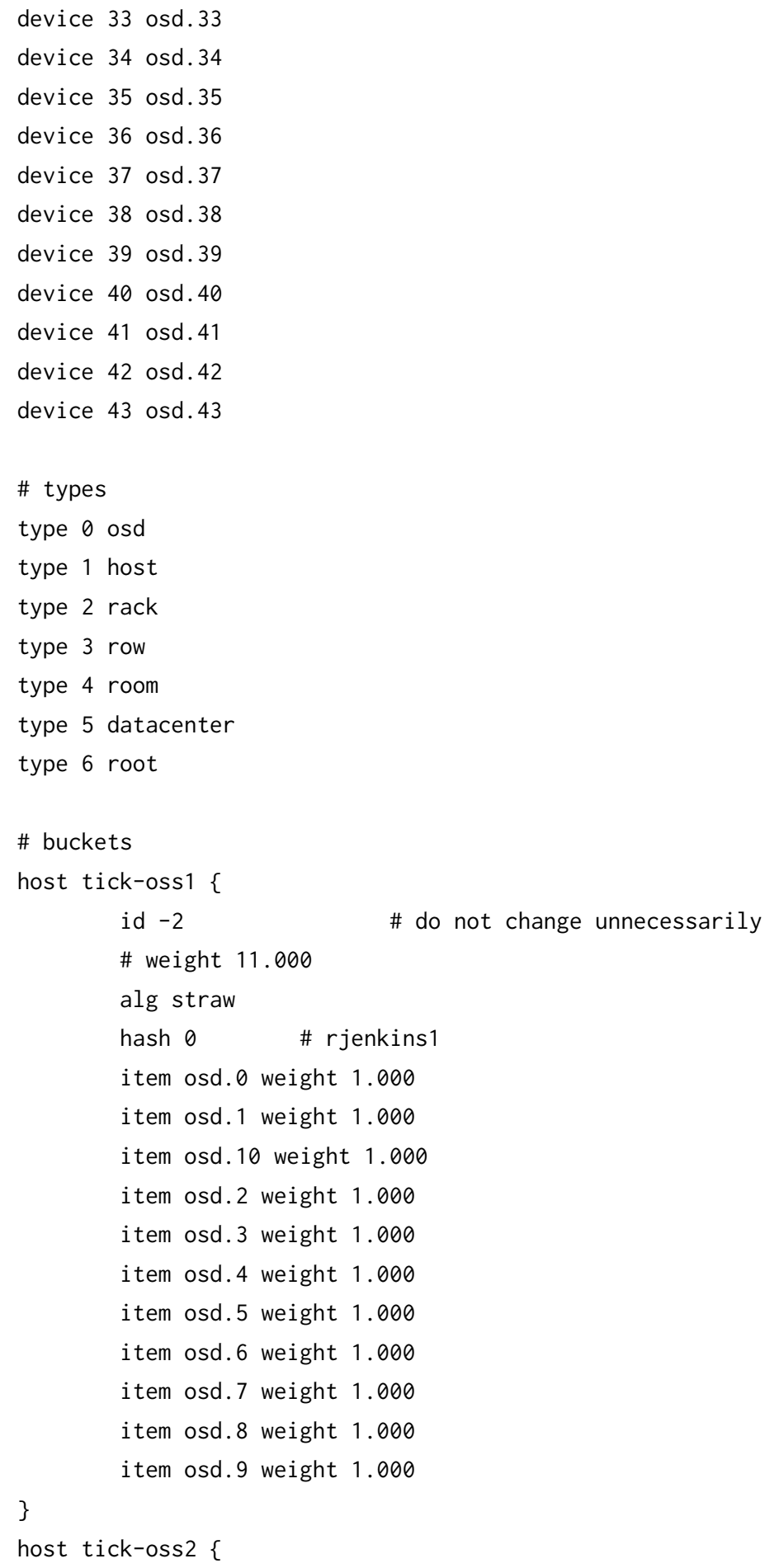




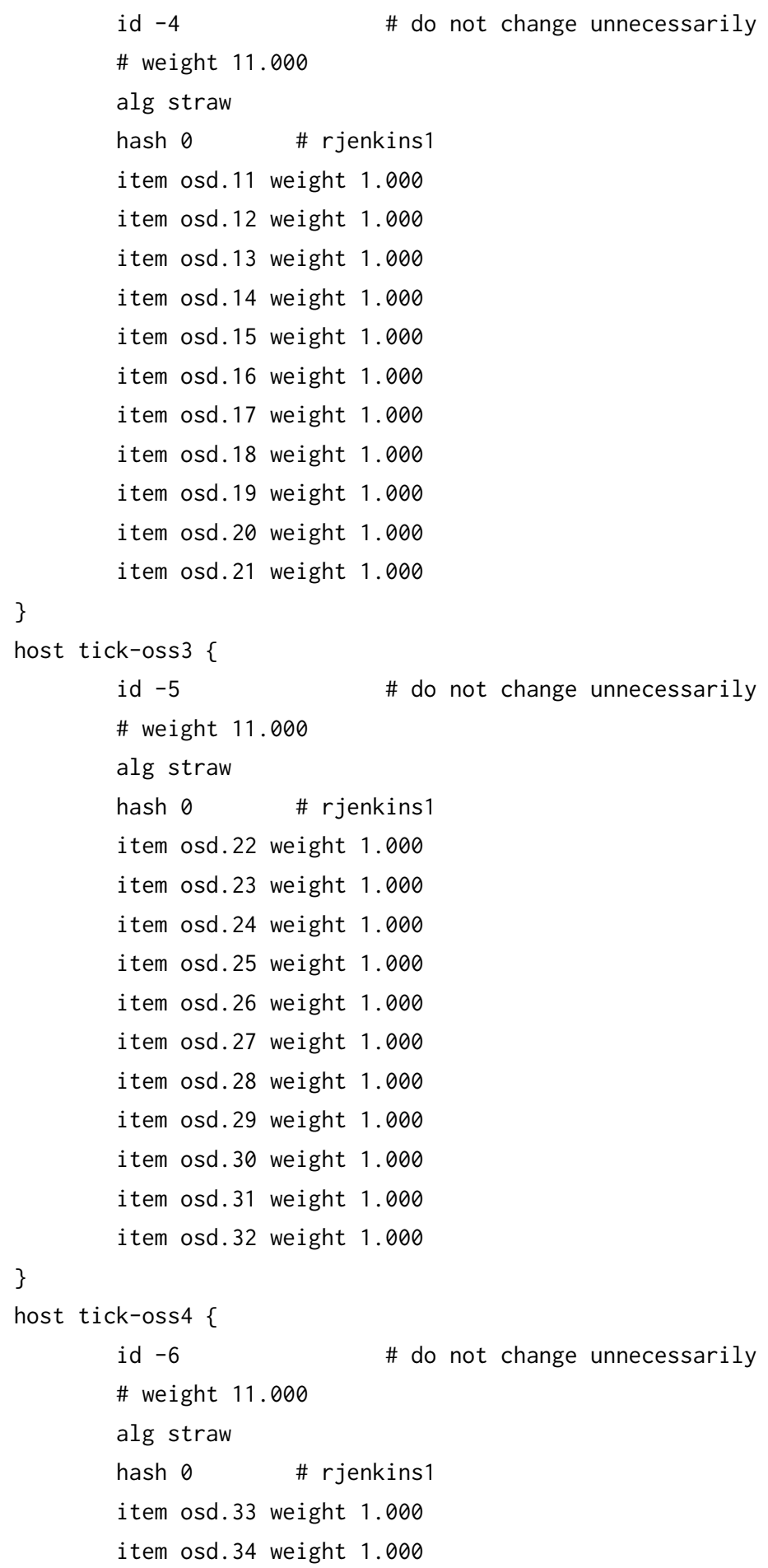




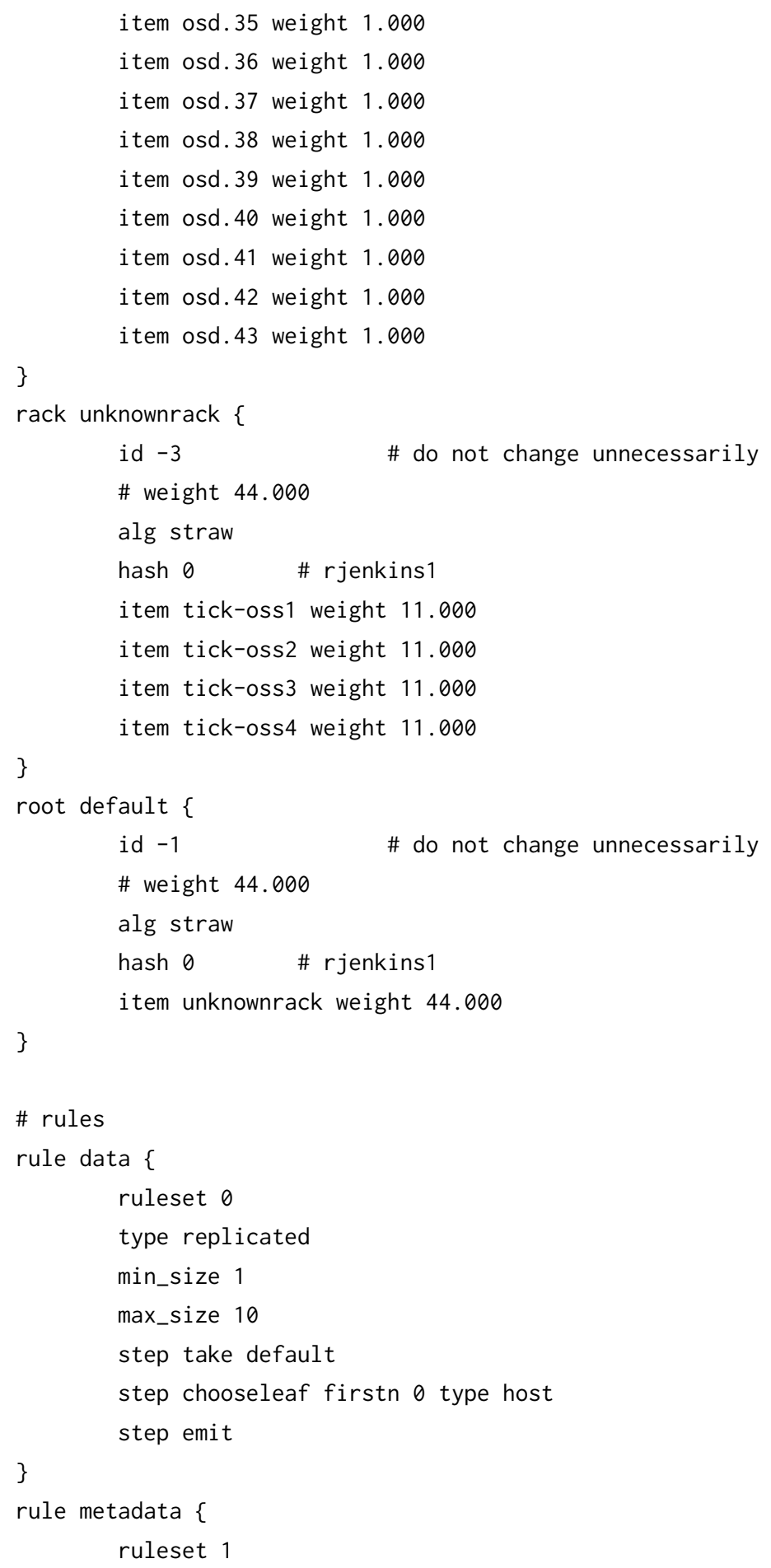




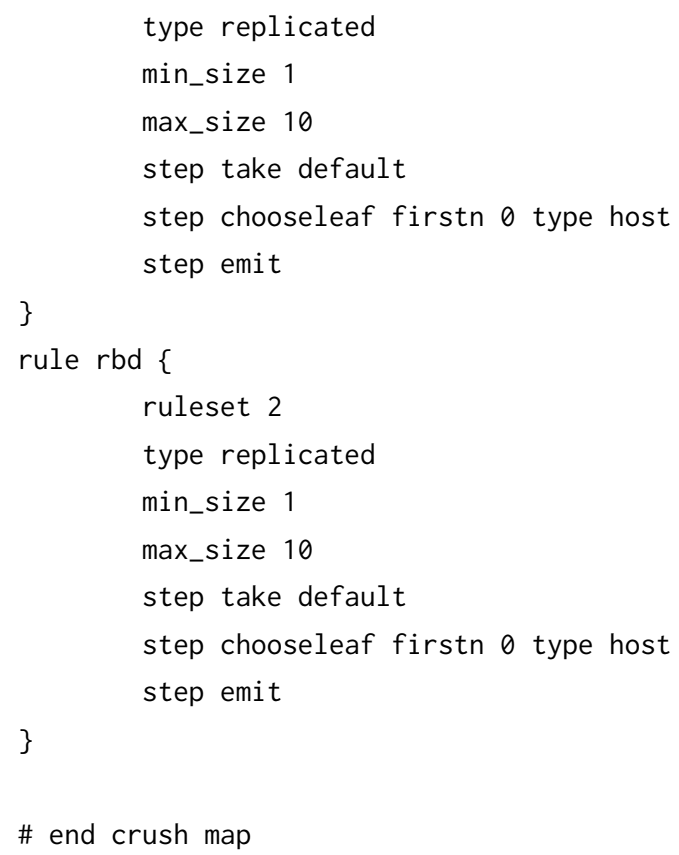

\section{Appendix D - Final ceph.conf}

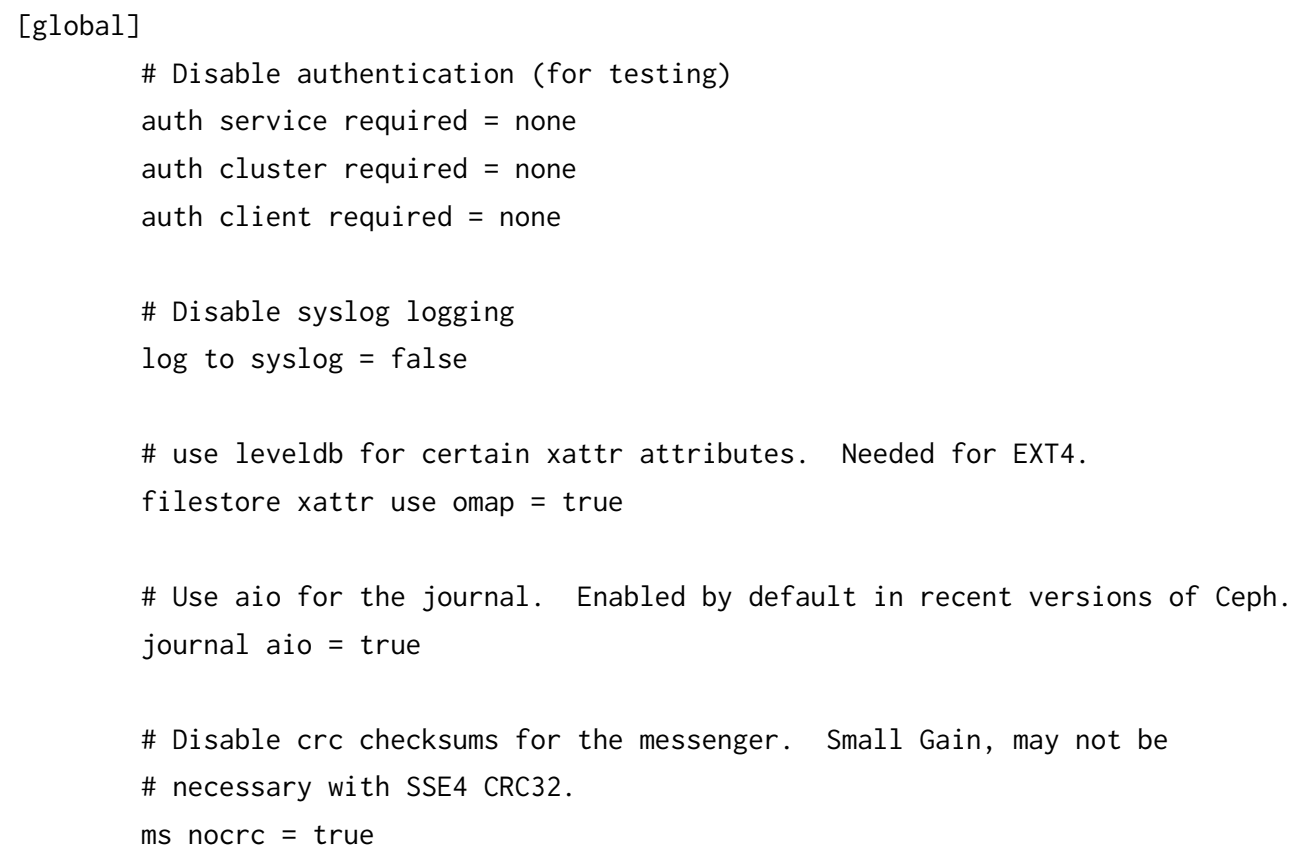




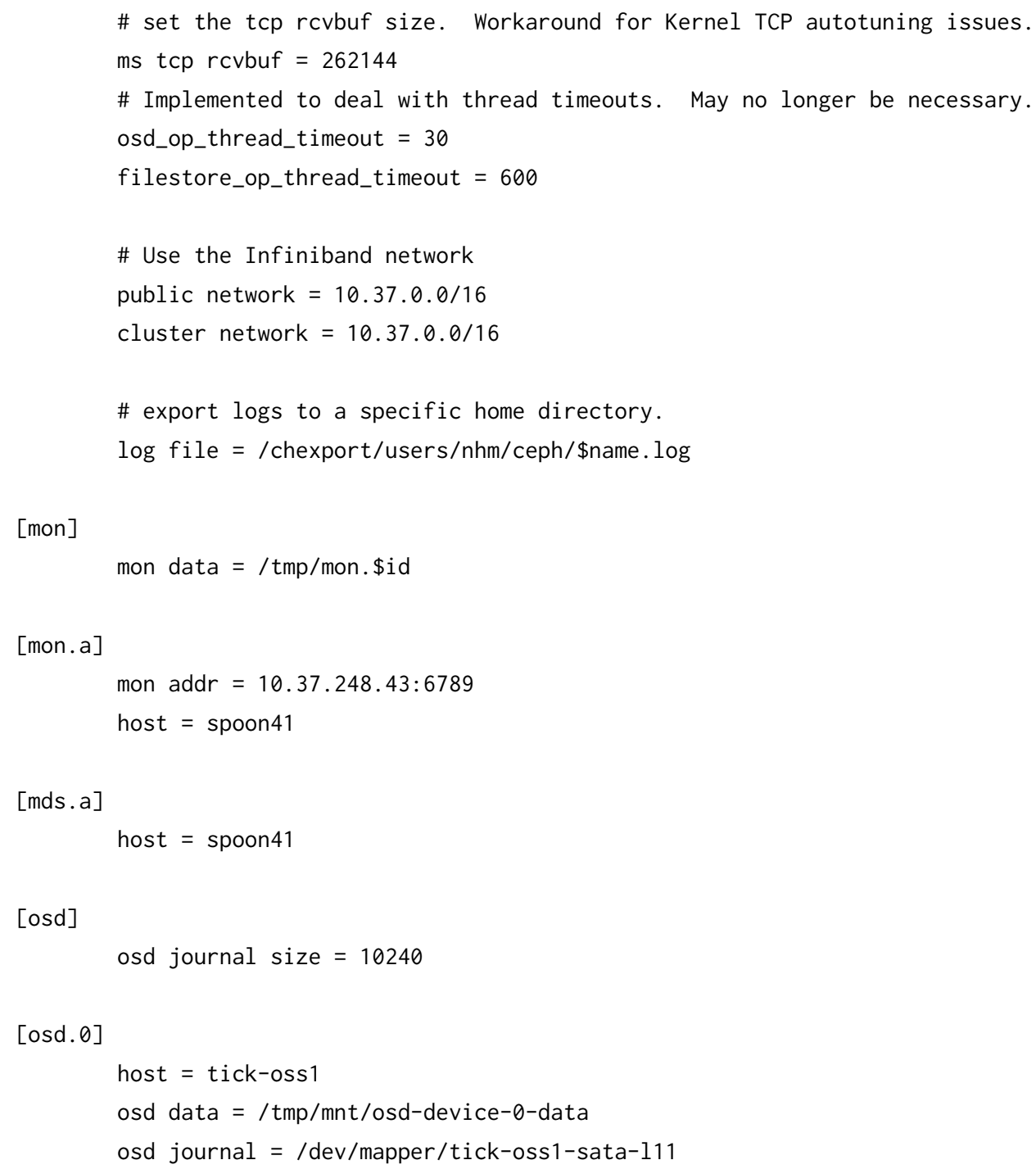

... Abbreviated for saving space

\section{Appendix E - Tuning Parameters}

For parameter sweep, here are the settings we iterated through. Some of these are "compound" settings where we increased or decreased multiple things in the ceph.conf file at the same time to reduce the number of permutations that would need to be tested. 


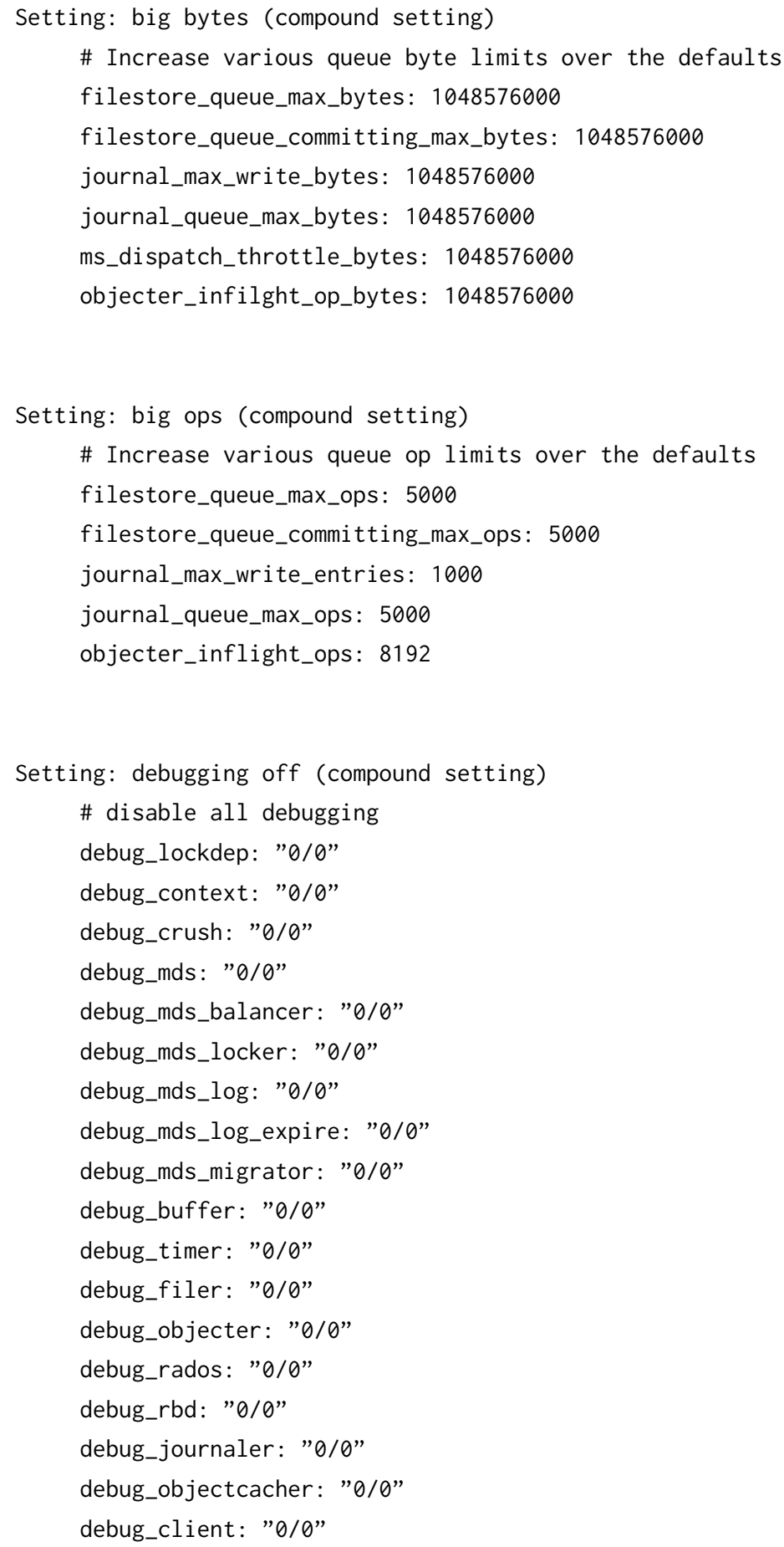




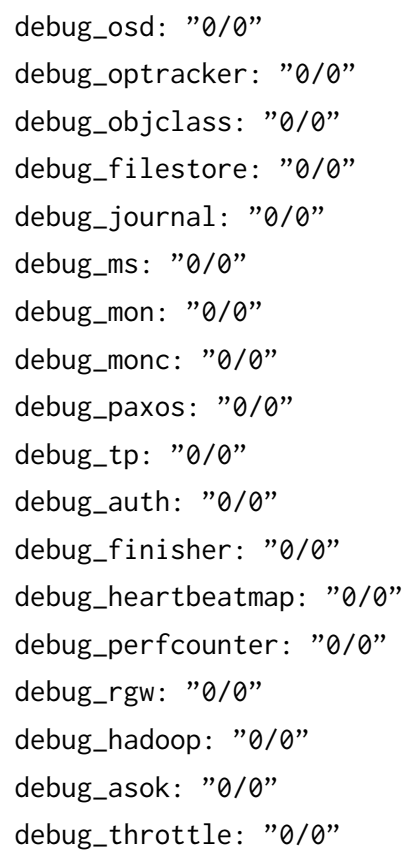


\# Enable asynchronous I0 for journal writes

Setting: ms_nocrc_true

\# Disable CRC checks in the messenger (IE for network transfers, but not for filestore)

Setting: osd_disk_threads

\# Number of threads used for background processes like scrubbing and snap trimming.

Setting: osd_op_threads

\# Number of threads to use for OSD Daemon operations

Setting: small bytes (compound setting)

\# Decrease various queue byte limits vs the defaults

filestore_queue_max_bytes: 10485760

filestore_queue_committing_max_bytes: 10485760

journal_max_write_bytes: 10485760

journal_queue_max_bytes: 10485760

ms_dispatch_throttle_bytes: 10485760

objecter_infilght_op_bytes: 10485760

Setting: small_ops (compound setting)

\# Decrease various queue op limits vs the defaults

filestore_queue_max_ops: 50

filestore_queue_committing_max_ops: 50

journal_max_write_entries: 10

journal_queue_max_ops: 50

objecter_inflight_ops: 128 